\title{
Dermatology and Vaccines: We Must Do Better
}

\author{
Shruti Agrawal, MD; Kenneth J. Tomecki, MD
}

V accines work. They are powerful tools that have saved millions of lives worldwide; however, a robust antivaccine movement has taken hold in the United States and worldwide despite overwhelming data in support of vaccination. In fact, vaccine hesitancy-the reluctance or refusal to vaccinate despite the availability of vaccines - was listed by the World Health Organization as one of the top 10 global health threats in $2019 .{ }^{1}$

Several vaccines have a role in dermatology, including the human papillomavirus (HPV) vaccine (Gardasil 9 [Merck Sharp \& Dohme Corp]), the herpes zoster vaccines (Zostavax [Merck Sharp \& Dohme Corp] and Shingrix [GlaxoSmithKline Biologicals]), and the measles-mumps-rubella vaccine, among others. These vaccinations are necessary for children and many adults alike, and they play a critical role in protecting both healthy and immunosuppressed patients.

Vaccine hesitancy is a growing threat to individual and public health that requires a response from all physicians. In our experience, dermatologists have been somewhat passive in advocating for vaccinations, possibly due to knowledge barriers or time constraints; however, this stance must change. Dermatologists must join the front lines in advocating for vaccinations, which are a proven and effective modality in promoting public health.

Dermatologists can employ the following practical tips to improve vaccination compliance among patients:

- Familiarize yourself with the Centers for Disease Control and Prevention immunization schedules and vaccination information sheets (https://www.cdc.gov /vaccines/hcp/vis/current-vis.html). Printed copies of informational handouts should be readily available to provide to patients in the office. The Centers for Disease Control and Prevention also offers tip sheets to guide conversations with patients (https://www.cdc.gov/vaccines /hcp/conversations/index.html).

- Prior to starting an immunosuppressive medication, confirm the patient's immunization status. You should know which vaccines are live (containing an attenuated pathogen) and which are inactivated. Live vaccines typically are not administered to immunosuppressed patients.

- Use electronic medical records to help provide reminders to prompt administration of any necessary vaccines.

- Know the facts, especially regarding purported vaccine controversies, and be able to cite data on vaccine safety and efficacy. For example, when having a conversation with a patient you could state that vaccination against $\mathrm{HPV}$, which can cause genital warts and certain cancers, has decreased the number of HPV infections by more than $70 \%$ in young women and $80 \%$ in teenaged girls. ${ }^{2}$ Cervical precancers were reduced by $40 \%$ in women vaccinated against HPV. Twelve years of monitoring data validates the safety and efficacy of the HPV vaccine-it is safe and effective, with benefits that outweigh any potential risks. ${ }^{2}$

- Tailor counseling based on the patient's age and focus on benefits that directly impact the patient. For example, consider showing young adults photographs of genital warts while educating them that the HPV vaccine can help prevent this kind of infection in the future. 
- Emphasize that vaccines are a routine part of comprehensive patient care and support this point by providing data and specific reasons for recommending vaccines. ${ }^{3}$ Avoid phrases such as, "Do you want the vaccine?" or "You could consider receiving the vaccine today," which can imply that the vaccine is not necessary.

- Offer vaccines in your office or provide clear printed informational sheets directing patients to nearby primary care clinics, infectious disease clinics, or pharmacies where vaccinations are offered.

- Consider using social media to promote the benefits of vaccination among patients.

The recent coronavirus disease 2019 pandemic has brought the topic of vaccination into the limelight while highlighting that rampant misinformation can lead to distrust of health care workers. Dermatologists, along with all physicians, should be trusted advisors and advocates for public health. In addition to being knowledgeable, dermatologists must remain open-minded in having conversations with skeptical patients. Physicians must take the time and effort to promote vaccinations-the health of patients and the general public depends on it.

\section{REFERENCES}

1. Akbar R. Ten threats to global health in 2019. World Health Organization website. https://www.who.int/emergencies/ten-threats-to -global-health-in-2019. Published March 21, 2019. Accessed November 11, 2020.

2. HPV vaccination is safe and effective. Centers for Disease Control and Prevention website. https://www.cdc.gov/hpv/parents/vaccinesafety. html. Updated April 29, 2019. Accessed November 11, 2020.

3. How to give a strong recommendation to adult patients who require vaccination. Medscape website. https://www.medscape.com/viewarticle 1842874. Published April 16, 2015. Accessed November 11, 2020. 